\title{
Gel de Chamomilla Recutita Para Reação de Pele em Paciente Submetido à Quimiorradioterapia: Relato de Caso
}

\author{
Chamomilla Recutita Gel for Skin Reactions of Patient Submitted to \\ Chemoradiation Therapy: Case Report
}

\section{Chamomilla Recutita Gel Para Reacciones Cutáneas en Pacientes Sometidos a Quimioradioterapia: Reporte de un Caso}

\author{
Elaine Barros Ferreira', Priscila de Souza Maggi Bontempo ${ }^{1,2}$, Marcos Antônio dos Santos²,
} Luis Felipe Oliveira e Silva², Samuel Ramalho Avelino², Paula Elaine Diniz dos Reis ${ }^{3}$

\begin{abstract}
RESUMO
O presente relato tem o objetivo de descrever o efeito do gel de Chamomilla recutita (C. recutita) em radiodermatite de paciente submetido à quimiorradioterapia — paciente do sexo masculino, com neoplasia de orofaringe, que fez uso do gel na concentração de 8,35\% na região irradiada. A partir da 16 a sessão, o paciente apresentou radiodermatite Grau I, segundo escore Radiation Therapy Oncology Group (RTOG), caracterizada por leve eritema da região irradiada. A graduação foi mantida até o final da radioterapia, com evolução dos sinais apresentados para hipercromia e descamação seca. Os benefícios clínicos da C. recutita na prevenção e no tratamento da radiodermatite evidenciam a importância de estudos experimentais, que possam corroborar os resultados descritos, considerando que a intervenção se mostrou uma alternativa de fácil implementação, fornecendo proteção e conforto ao paciente.
\end{abstract}

DESCRITORES: Radiodermatite. Quimiorradioterapia. Chamomilla. Estomaterapia.

\begin{abstract}
This report aims at describing the effect of Chamomilla recutita (C. recutita) gel in radiodermatitis of a patient submitted to chemoradiotherapy - male patient, with oropharyngeal cancer, that used the gel in the $8.35 \%$ concentration in the irradiated region. From the $16^{\text {th }}$ session, the subject presented Grade I radiodermatitis, according to the RTOG score, characterized by mild erythema in the irradiated region. Graduation was maintained until the end of radiotherapy, with an increase of the signals presented for hyperpigmentation and dry desquamation. The clinical benefits of $C$. recutita in the prevention and treatment of radiodermatitis show the importance of experimental studies that may corroborate the described results, considering that the intervention proved to be an alternative of easy implementation, thus providing protection and comfort to the patient.
\end{abstract}

DESCRIPTORS: Radiodermatitis. Chemoradiotherapy. Chamomilla. Stomatherapy.

Trabalho desenvolvido pelo Grupo de Pesquisa Laboratório Interdisciplinar de Pesquisa Aplicada à Prática Clínica em Oncologia, vinculado ao CNPq. 1Programa de Pós-Graduação em Enfermagem da Universidade de Brasília (PPGENF/UnB) - Brasília (DF), Brasil.

${ }^{2}$ Centro de Alta Complexidade em Oncologia do Hospital Universitário de Brasília (CACON/HUB) - Brasília (DF), Brasil.

${ }^{3}$ Departamento de Enfermagem da Faculdade de Ciências da Saúde da Universidade de Brasília (UnB) - Brasília (DF), Brasil.

Endereço para correspondência: Elaine Barros Ferreira - Universidade de Brasília, Campus Universitário Darcy Ribeiro - Asa Norte - CEP: $70910-900$ -

Brasília (DF), Brasil. E-mail: elaine.barrosf@gmail.com

Artigo recebido em: 24/09/2015 - Aceito para publicação em: 25/07/2016 


\section{RESUMEN}

Este reporto tiene como objetivo describir el efecto del gel de Chamomilla recutita (C. recutita) en la radiodermatitis en pacientes sometidos a quimioradioterapia. Paciente del género masculino, con el cáncer de la orofaringe. El paciente ha hecho uso del gel a una concentración de 8,35\% en la región irradiada. Desde el $16^{\circ}$ período de sesiones, él ha presentado la radiodermatitis del Grado I, de acuerdo con la puntuación RTOG, caracterizada por eritema leve de la región irradiada. La graduación se mantuvo hasta el final de la radioterapia, con un aumento de las señales presentadas a la hiper-pigmentación y descamación seca. Los beneficios clínicos de C. recutita en la prevención y en el tratamiento de la radiodermatitis muestran la importancia de estudios experimentales que puedan corroborar los resultados descritos, considerando que la intervención se demostró como una alternativa fácil de implementar, proporcionando la protección y la comodidad para el paciente.

DESCRIPTORES: Radiodermatitis. Quimioradioterapia. Chamomilla. Estomaterapia.

\section{INTRODUÇÃO}

O carcinoma de células escamosas de cabeça e pescoço localmente avançado tem como tratamento padrão a quimiorradioterapia com cisplatina ${ }^{1}$. Embora essa associação terapêutica seja benéfica na terapia antineoplásica, ela pode aumentar a incidência e a severidade de reações adversas - como a radiodermatite — , sendo importante seu manejo adequado e considerando os impactos negativos das reações no tratamento e na qualidade de vida do paciente ${ }^{2}$.

No que diz respeito à prevenção e ao tratamento da radiodermatite, as intervenções aplicadas são inconsistentes, havendo discordância entre as diversas recomendações oferecidas aos pacientes ${ }^{3}$.

A aplicação tópica da Chamomilla recutita (C. recutita) tem sido demonstrada em estudos quanto aos seus benefícios no tratamento de reações da pele - como dermatite, eczema, radiodermatite e cicatrização de feridas ${ }^{4}$ bem como sua propriedade anti-inflamatória, atribuída aos seus constituintes - como camazuleno, $\alpha$-bisabolol e apigenina ${ }^{5}$.

O presente relato teve por objetivo descrever o efeito do gel de $C$. recutita em radiodermatite de paciente submetido à quimiorradioterapia.

\section{MÉTODOS}

Trata-se de um relato de caso realizado no período de dezembro de 2014 a fevereiro de 2015.
O relato descreve o uso tópico do gel de C. recutita por paciente com neoplasia de orofaringe submetido à radioterapia. O gel, manipulado em farmácia de manipulação especializada, composto pelo extrato da flor de C. recutita em meio aquoso, apresenta concentração de 8,35\%, sendo esta utilizada como intervenção em estudo de fase II, conduzido para determinação da dose de segurança do gel nesses pacientes, conforme protocolo de estudo registrado no Clinical Trials (NCT02249884).

O estudo foi aprovado pelo Comitê de Ética em Pesquisa da Faculdade de Ciências da Saúde (CEP/FS), processo CAAE no 24692813.6.0000.0030, conforme preconiza a Resolução 466/12 do Conselho Nacional de Saúde. Obtiveram-se, ainda, o termo de consentimento livre e esclarecido do paciente e a assinatura da autorização do termo de uso de imagem. Os dados foram coletados a partir de entrevista, prontuário do paciente, avaliação e acompanhamento diário da evolução da reação local.

\section{RESULTADOS}

Paciente do sexo masculino, 48 anos, com neoplasia de orofaringe, submetido à radioterapia, concomitante à quimioterapia com cisplatina, a cada 21 dias. Paciente nega tabagismo e refere baixo consumo de bebidas alcoólicas.

Realizou radioterapia conformacional tridimensional (3D-CRT) com feixes de fótons de $6 \mathrm{MV}$, recebendo dose de 45 Gy, em 25 frações, nas cadeias de drenagem eletivas e um complemento de 25,2 Gy, em 14 frações, no volume tumoral macroscópico, totalizando 70,2 Gy. 
Concomitante ao início da radioterapia, o paciente aplicou, sem interrupções, três vezes ao dia, gel de $C$. recutita a $8,35 \%$ na região irradiada. As orientações referentes aos cuidados ao longo do tratamento - evitar exposição ao sol da área irradiada, hidratação com consumo de $2 \mathrm{~L}$ de líquidos diariamente, evitar extremos de temperatura, não utilizar lâmina de barbear para retirada dos pelos, limpeza da região com solução saponácea e não aplicar outro produto sobre a área irradiada - foram oferecidas pela enfermeira, no momento da consulta de Enfermagem, que antecedeu o início do tratamento. Dentre as orientações quanto à aplicação do produto, o paciente era informado quanto à forma e quantidade de aplicação e quanto à remoção do produto da pele antes de cada sessão de radioterapia. Foi realizado acompanhamento diário para avaliação da pele e fotografada semanalmente a região irradiada para acompanhamento da evolução da reação cutânea.

Paciente iniciou a radioterapia com pele íntegra, sem qualquer alteração (Figura 1). A partir da 16a sessão, apresentou radiodermatite Grau I, segundo escore RTOG para toxicidade aguda ${ }^{6}$, caracterizada por leve eritema em região irradiada (Figura 2). Ao final da segunda fase da radioterapia, o paciente evoluiu com hipercromia da área irradiada e áreas de descamação seca, indicativos de radiodermatite Grau I (Figura 3), mantendo, portanto, a mesma graduação

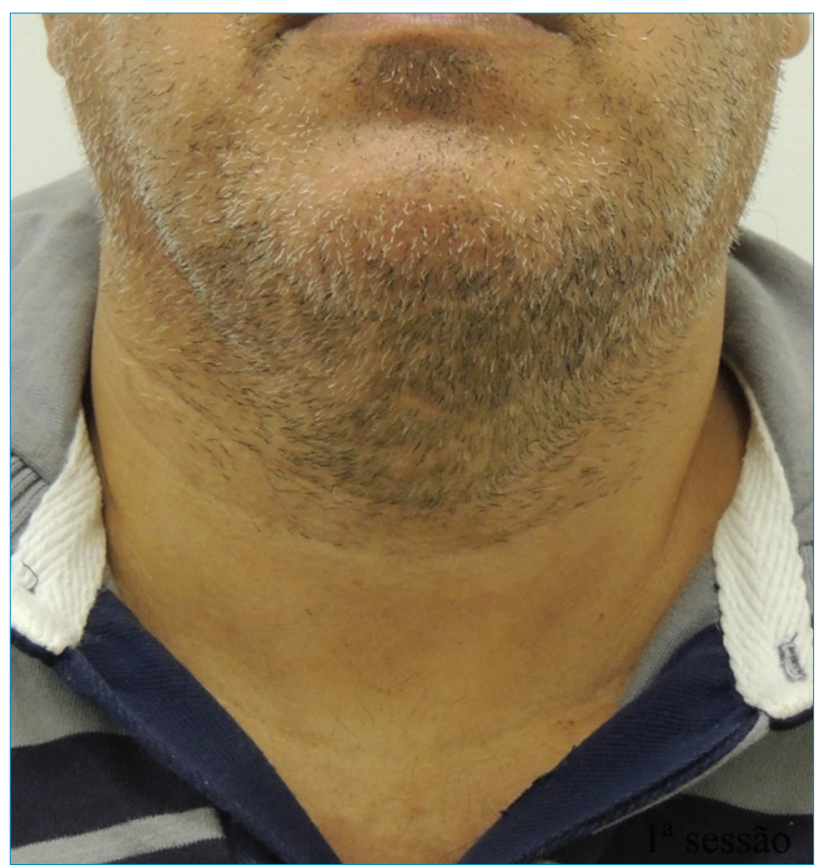

Figura 1. Pele íntegra e sem sinais de alteração ao início da radioterapia. $1^{\text {a }}$ sessão de radioterapia. Brasília, 2015. para reação cutânea, ainda que com evolução dos sinais apresentados, ao longo de todo tratamento. $\mathrm{O}$ paciente referiu sensação de alívio, conforto e frescor na região irradiada ao utilizar o produto.

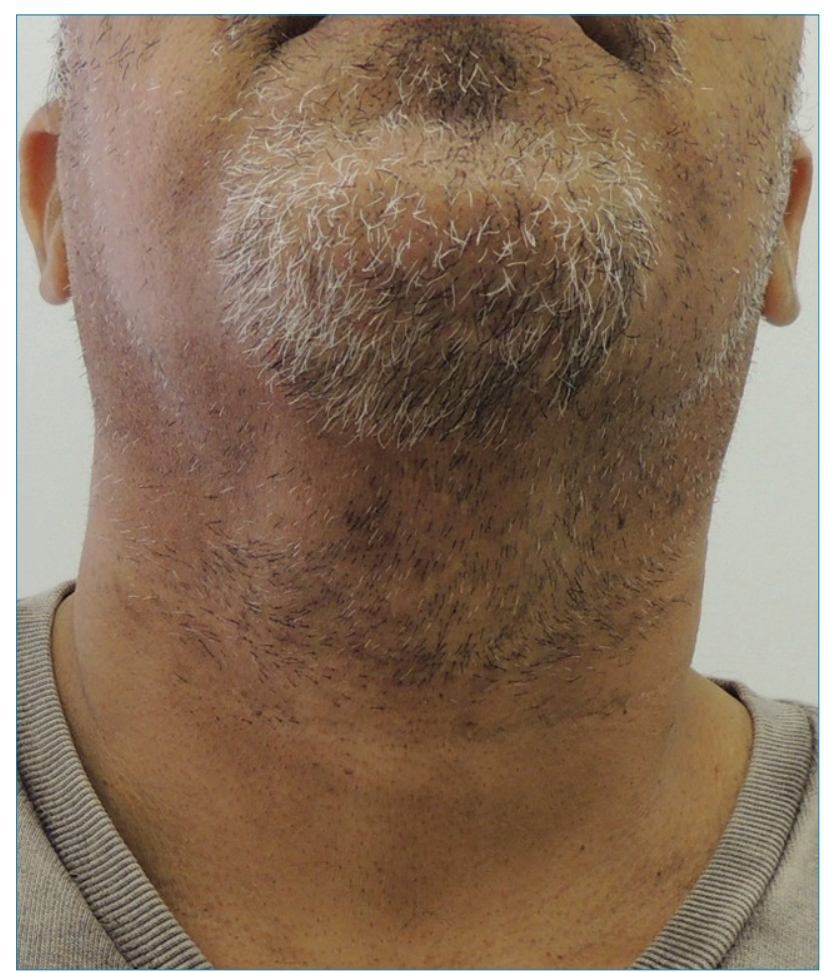

Figura 2. Início do eritema em região irradiada. $16^{\mathrm{a}}$ sessão de radioterapia. Brasília, 2015.

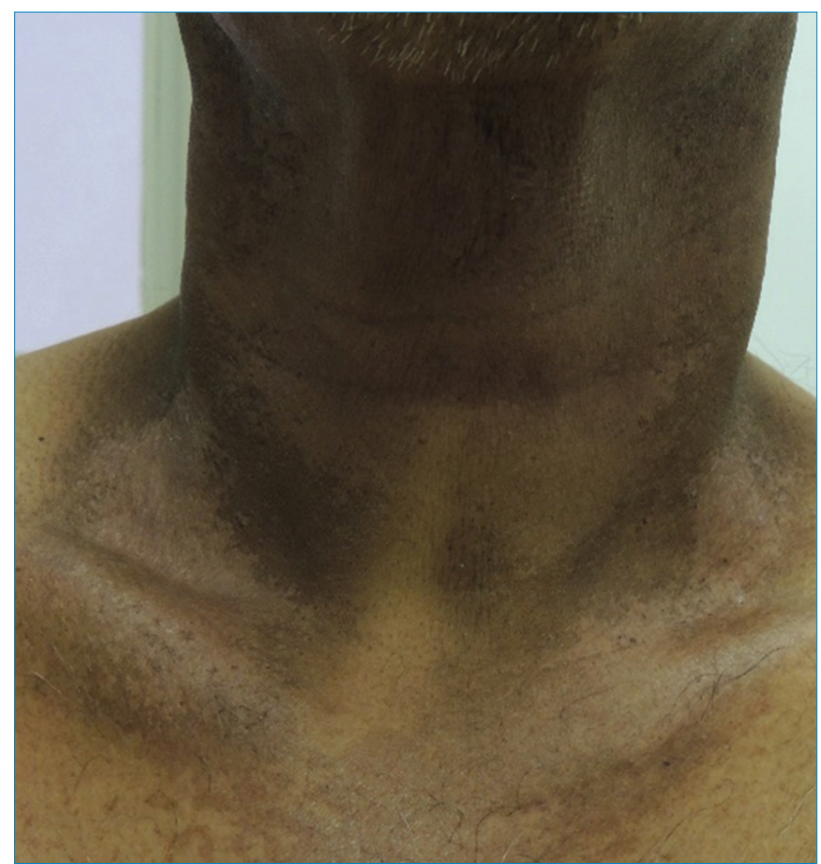

Figura 3. Região irradiada com presença de hipercromia e descamação seca. 36 sessão de radioterapia. Brasília, 2015. 


\section{DISCUSSÃO}

As doses total e fracionada administradas no paciente durante a radioterapia associadas à quimioterapia concomitante são fatores extrínsecos que influenciam na intensidade da reação cutânea ${ }^{3}$. Tais fatores geralmente induzem uma intensidade reacional superior à observada ao longo do tratamento no paciente acompanhado, o que pode sugerir um benefício ocasionado pelo uso diário do gel de C. recutita.

Comumente, identificam-se hipercromia e descamação seca na região irradiada após duas a quatro semanas de tratamento, ao se atingir 20 Gy de dose cumulativa ${ }^{7}$. No entanto, na $16^{\mathrm{a}}$ sessão da primeira fase de tratamento, quando o paciente já havia recebido $28,8 \mathrm{~Gy}$, sua pele apresentava apenas sinais de eritema leve.

O benefício observado é consistente com a atividade anti-inflamatória da C. recutita, que inibe a formação de mediadores inflamatórios ${ }^{8}$ - como histamina e serotonina -, que são secretados a partir da resposta inflamatória instaurada com o desenvolvimento da radiodermatite ${ }^{7}$. Duas formulações - enriquecidas com apigenina, flavonoide constituinte da C. recutita - foram avaliadas para potencial utilização como agentes anti-inflamatórios tópicos, indicando que a apigenina é capaz de inibir o processo inflamatório ${ }^{4}$.

A hidratação da área irradiada é geralmente considerada o método ideal para prevenir e minimizar reações de pele induzidas por radiação ${ }^{9}$. Em estudo pré-clínico, o uso tópico da apigenina, com o mesmo propósito anti-inflamatório, foi avaliado em ratos com dermatite de contato aguda, resultando em importante redução da perda de água transepidérmica, melhorando a função da barreira de permeabilidade da epiderme e a hidratação do estrato córneo ${ }^{10}$.

Os benefícios clínicos da C. recutita na prevenção e no tratamento da radiodermatite devem ser considerados, evidenciando a importância de novos estudos, de caráter experimental, que possam corroborar com os resultados descritos e forneçam mais evidências sobre sua eficácia e efetividade, considerando que a intervenção se mostrou uma alternativa de fácil implementação, fornecendo especial proteção e conforto ao paciente.

\section{CONCLUSÃO}

Sugere-se que a aplicação tópica do gel de C. recutita, no caso clínico apresentado, possa ter viabilizado a redução da intensidade da reação local, o atraso no desenvolvimento dos primeiros sinais da reação cutânea, o retardo na evolução da reação Grau I e exercido efeito protetor no que concerne à severidade da reação. Destaca-se também a intervenção ter sido avaliada positivamente pelo paciente no aspecto sensorial.

\section{REFERÊNCIAS}

1. Giralt J, Trigo J, Nuyts S, Ozsahin M, Skladowski K, Hatoum G, et al. Panitumumab plus radiotherapy versus chemoradiotherapy in patients with unresected, locally advanced squamous-cell carcinoma of the head and neck (CONCERT-2): a randomised, controlled, open-label phase 2 trial. Lancet Oncol. 2015;16(2):221-32.

2. Imai T, Matsuura K, Asada Y, Sagai S, Katagiri K, Ishida E, et al. Effect of HMB/Arg/GIn on the prevention of radiation dermatitis in head and neck cancer patients treated with concurrent chemoradiotherapy. Jpn J Clin Oncol. 2014;44(5):422-7.

3. Chan RJ, Webster J, Chung B, Marquart L, Ahmed M, Garantziotis S. Prevention and treatment of acute radiation-induced skin reactions: a systematic review and meta-analysis of randomized controlled trials. BMC Cancer. 2014;14(53):1-19.

4. Arsic' I, Tadic' V, Vlaovic' D, Homšek I, Vesic' S, Isailovic' $\mathrm{G}$, et al. Preparation of novel apigenin-enriched, liposomal and non-liposomal, antiinflammatory topical formulations as substitutes for corticosteroid therapy. Phytother Res. 2011;25(2):228-33.

5. Shimelis ND, Asticcioli S, Baraldo M, Tirillini B, Lulekal E, Murgia $\vee$. Researching accessible and affordable treatment for common dermatological problems in developing countries. An Ethiopian experience. Int J Dermatol. 2012; 51(7):790-5.

6. Cox JD, Stetz J, Pajak TF. Toxicity criteria of the Radiation Therapy Oncology Group (RTOG) and the European Organization for Research and Treatment of Cancer (EORTC). Int J Radiat Oncol Biol Phys. 1995;31(5):1341-6.

7. McQuestion M. Evidence-based skin care management in radiation therapy: clinical update. Semin Oncol Nurs. 2011;27(2):e1-17.

8. Srivastava JK, Shankar E, Gupta S. Chamomile: a herbal medicine of the past with bright future. Mol Med Rep. 2010;3(6):895-901.

9. Salvo N, Barnes E, Van Draanen J, Stacey E, Mitera G, Breen $D$, et al. Prophylaxis and management of acute radiationinduced skin reactions: a systematic review of the literature. Curr Oncol. 2010;17(4):94-112.

10. Man MQ, Hupe M, Sun R, Man G, Mauro TM, Elias PM. Topical apigenin alleviates cutaneous inflammation in murine models. Evid Based Complement Alternat Med. 2012;2012:1-7. 